\title{
CARACTERIZACIÓN PRODUCTIVA Y TERRITORIAL DE LAS GANADERÍAS DE BOVINO DE LECHE EN CANTABRIA, SEGÚN EL CONTENIDO DE FORRAJE VERDE EN LA DIETA (PÓSTER)
}

\author{
$\underline{\text { Francisca Ruiz Escudero }}^{a *}$, Ibán Vázquez González ${ }^{b}$, Ana Villar Bonet ${ }^{\mathrm{a}}$ y Juan Busqué Marcos ${ }^{\mathrm{a}}$ \\ a Centro de Investigación y Formación Agrarias (CIFA). Consejería de Desarrollo Rural, Ganadería, \\ Pesca, Alimentación y Medio Ambiente del Gobierno de Cantabria (Muriedas, ruiz_fr@cantabria.es; \\ villar_am@cantabria.es; busqué_jc@cantabria.es). \\ ${ }^{b}$ Universidade de Santiago de Compostela. Escola Politécnica Superior de Enxeñaría (Dpto. Economía \\ Aplicada(Lugo, iban.vazquez.gonzalez@usc.es).
}

\begin{abstract}
Resumen
En los últimos años, están apareciendo en el mercado leche en la que se indica "de pastoreo" o "de pasto", debido al interés creciente de la sociedad hacia productos más saludables y respetuosos con el medioambiente. El objetivo de este trabajo es profundizar en la relación entre las características territoriales de las ganaderías de bovino de leche y su manejo alimentario. Se clasificaron 99 ganaderías en función del porcentaje de forraje verde (FV) en la dieta -de primavera- de las vacas en lactación (No pasto $<25 \% \mathrm{FV}$; Pasto $\geq 25 \% \mathrm{FV}$ ). Como variables se incluyeron el porcentaje de materia seca de los distintos componentes de la dieta, las principales características productivas y la información territorial contenida en la base de datos oficial SIA (Sistema integrado de ayudas) para 41 variables territoriales. Las ganaderías pertenecientes al modelo en base a pasto $(\geq 25 \% \mathrm{FV})$ constituyen el $33 \%$ y presentan una menor dimensión productiva, carga ganadera y consumo de concentrado, con una mayor dedicación al pastoreo. Además, presentan mayor altitud de las parcelas, parcelas de mayor dimensión, más homogéneas y con mejor geometría, pero también un suelo de baja capacidad de uso agrícola. Este trabajo abre la posibilidad a analizar el potencial productivo y territorial de Cantabria para la producción de leche en base a pasto, como estrategia de valorización de los sistemas extensivos de producción de leche ligada al territorio.
\end{abstract}

Palabras clave: Bovino, leche de pasto, forraje verde, variables territoriales, Cantabria.

\section{Introducción y objetivos}

El bovino de leche es un sector estratégico para Cantabria por su importancia económica, sociocultural y por sus implicaciones en el territorio. Los últimos datos del sector bovino de leche entre 2016-2020 (MAPA, 2020) muestran la tendencia descendente en el número de ganaderías (-22\%), tanto a nivel nacional como en Cantabria, y la continuación del proceso de concentración productiva, desapareciendo las de menor dimensión inversamente a la expansión de las ganaderías más grandes. En este sentido, la estructura productiva del bovino de leche en Cantabria pone de manifiesto la mayoritaria presencia de la pequeña y mediana ganadería, la importancia del uso de forraje verde (en adelante, FV) en la alimentación de los animales y la relevancia de la actividad de pastoreo, fomentando el mantenimiento del paisaje en esta comunidad, así como contribuyendo a mitigar el cambio climático, en consonancia con el enfoque marcado por la UE (PVE) para la futura PAC. Además, los pastos herbáceos tienen una destacada presencia en Cantabria, pues representan un $44 \%$ de la superficie agraria útil (CIFA, 2007), con una de las mayores productividades forrajeras europeas (Huyghe et al., 2014; Smit et al., 2008).

El objetivo de este trabajo estriba en profundizar en el análisis de las principales características productivas y territoriales de las ganaderías de bovino de leche, en función de la importancia de la inclusión de forraje verde en la dieta de las vacas en lactación. Con ello se pretende contribuir al estudio de su estructura productiva, al tiempo que se visibiliza su gestión y manejo en base a pasto, como estrategia de valorización de los sistemas extensivos de producción de leche ligada al territorio.

\section{Metodología}

\section{Información sobre el manejo alimentario}

La información sobre el sistema de alimentación (la dieta concreta de las vacas en lactación a lo largo del año) y otras características productivas, se obtuvo de encuestas llevadas a cabo en la propia explotación (fuente primaria de datos). Se trabajó con 25 ganaderías de leche de Cantabria colaboradoras dentro de un proyecto de investigación nacional INIA (RTA2014-0086-C03), entrevistadas en 2016. La selección de las granjas se llevó a cabo buscando la mayor diversidad posible de manejos productivos y sistemas de alimentación (Villar et al., 2021a). Por otra parte, se trabajó con los datos de 86 encuestas de ganaderías de vacuno de leche en el marco de un proyecto de tesis doctoral sobre el sector bovino en Cantabria (convocatoria FPI INIA 2015, García et al., 2020), realizadas entre noviembre de 2016 y febrero de 2017. Esta selección de las granjas se realizó en función de la producción de leche en la campaña 2015-2016, 
mediante un muestreo aleatorio estratificado de afijación de mínima varianza de Neyman, para un error de muestreo del $5 \%$ y nivel de confianza del $95 \%$.

\section{Información sobre la estructura territorial}

La información territorial se obtuvo de fuentes de datos secundarios procedentes del Sistema integrado de ayudas (SIA), correspondientes al año 2015, para todas las ganaderías de bovino de leche en Cantabria. Con la función ClassStat del paquete SDMTools del programa informático R, se crearon una serie de métricas espaciales (McGarigal, 2017), del que se elaboraron seis archivos de información con un total de 89 variables territoriales.

\section{Creación de la base de datos unificada}

Tras calcular las métricas espaciales se cruzó la información obtenida en las encuestas directas con la información territorial que se tenía de cada una de las ganaderías encuestadas en una única base de datos (primarios y secundarios). Debido a la ausencia de la información territorial para 5 municipios de Cantabria (12 explotaciones), el número final de explotaciones incluidas en el estudio se redujo a 99.

\section{Estimación de la dieta}

La composición de la dieta de las vacas en lactación en primavera se expresó en términos de porcentaje de ingesta de materia seca (MS) de cada componente de la ración por vaca y día, es decir: \% hierba fresca (consumido ya sea en pesebre o en pastoreo), $\%$ ensilado de hierba, $\%$ ensilado de maíz, \% forraje seco (suma de la materia seca ingerida como heno, paja, alfalfa, etc.) y \% concentrado.

En las ganaderías NFV se calculó el porcentaje de MS de cada componente de la ración diaria en función de la información suministrada por la ganadería. En el caso de ganaderías en las que las vacas consumían $\mathrm{FV}$, bien en pastoreo o bien en pesebre, fue necesario estimar teóricamente esa ingesta en función de los requerimientos teóricos de energía neta total del ganado lechero y aplicando las ecuaciones de predicción del National Research Council (NRC, 2001).

\section{Selección de variables territoriales}

De la información territorial suministrada con las métricas espaciales, se seleccionan 41 variables con las que trabajar, atendiendo a criterios de calidad de la información (mínimo número de explotaciones con datos ausentes) y representatividad (variables más importantes). Las variables seleccionadas contienen información relativa a la altitud (4 variables), estructura de la superficie (7 variables), métricas espaciales (11 variables), estructura de la superficie a $\leq 1 \mathrm{~km}$ (11 variables), calidad del suelo para uso agrícola (2 variables), presencia de ganaderías vecinas (3 variables) y potencial superficie (3 variables).

\section{Análisis estadísticos descriptivos y ANOVA}

Las ganaderías se clasificaron en dos grupos en función del porcentaje de forraje verde, sobre la materia seca total, en la dieta de primavera de las vacas en lactación: ganaderías sin forraje verde (NFV, < 25\% FV) y ganaderías con forraje verde ( $\mathrm{FV}, \geq 25 \%$ ); este umbral se marcó en función de los resultados obtenidos en un estudio anterior (Villar et al., 2021b). Una vez clasificadas las ganaderías según el sistema de alimentación, se llevaron a cabo análisis estadísticos descriptivos (valores medios y número de casos) y análisis de la varianza (ANOVA), con el objeto de llevar a cabo la caracterización tanto productiva como de la estructura territorial.

\section{Resultados}

\subsection{Relación entre dieta y sistema productivo}

\section{Caracterización productiva}

Los resultados ponen de manifiesto la existencia de diferencias muy significativas entre los dos estratos de ganaderías para todos los parámetros indicados, tanto respecto a la dieta como en relación a las variables productivas (Cuadro 1). En cuanto a las características productivas, las explotaciones FV tienen, inversamente a las ganaderías NFV, una menor dimensión territorial (SAU) y ganadera (UGM leche) (casi tres veces inferior), una menor carga ganadera, una producción anual de leche casi cuatro veces inferior y menor consumo de concentrado, mientras las horas diarias dedicadas a pastoreo son, sustancialmente, mayores. Por otro lado, cabe señalar la similitud en el consumo de concentrado entre ambos estratos que, de media y para las ganaderías incluidas en el análisis, asciende a 9,82 kg/vaca/día. Destacan, claramente, dos variables por su marcada diferencia entre los dos estratos, las UGM y la producción anual de leche.

Respecto a la dieta, y como era de esperar, el forraje verde es el componente más diferencial, sin embargo el contenido medio de concentrado, aun siendo estadísticamente diferente, es alto en ambos grupos. Ambos son los componentes mayoritarios, en la dieta de los rebaños de bovino de leche en Cantabria, destacando, en primer lugar, el pienso con un porcentaje medio de 44,46\% y, en segunda posición, el FV con un 18,85\%.

De forma clara y para las ganaderías NFV, este concentrado es el componente mayoritario $(49,1 \%)$, mientras que en las ganaderías FV, el elemento principal es el forraje verde, constituyendo de media un 
$52 \%$ de la materia seca. En menor medida y en orden decreciente para ambos grupos, se sitúan otros componentes como el forraje seco, silo de hierba y silo de maíz, este último minoritario en las dietas FV.

Cuadro 1. Características productivas y composición porcentual de la dieta de primavera, según tipo de ganadería FV ( $25 \%$ FV) y NFV $(<25 \%$ FV), años 2016-2017

\begin{tabular}{|l|c|c|c|}
\hline & Grupo NFV & Grupo FV & \multirow{2}{*}{ Total } \\
\cline { 2 - 3 } & $<25 \% \mathrm{FV}^{(1)}$ & $\geq 25 \% \mathrm{FV}$ & \\
\hline \multicolumn{1}{|c|}{ Variables productivas (valores medios) } & $\mathrm{N}=66$ & $\mathrm{~N}=33$ & $\mathrm{~N}=99$ \\
\hline $\mathrm{SAU}^{(2)}$ leche (ha) & 43,15 & 29,14 & $38,48 * *(4)$ \\
\hline $\mathrm{UGM}^{(3)}$ leche (ud) & 162,16 & 59,51 & $127,94 * * *$ \\
\hline Carga ganadera (UGM leche ha) & 3,87 & 2,37 & $3,37 * * *$ \\
\hline Producción anual (litros) & 1.049 .141 & 294.746 & $797.676 * * *$ \\
\hline Consumo concentrado (Kg pienso/vaca/día) & 10,93 & 7,62 & $9,82 * * *$ \\
\hline Horas pastoreo día & 1,05 & 6,57 & $2,89 * *$ \\
\hline$\quad$ Composición dieta (\% respecto a la MS total) & & & \\
\hline Silo de hierba & 16,95 & 2,61 & $12,17 * * *$ \\
\hline Silo de maíz & 14,03 & 0,56 & $9,54 * * *$ \\
\hline Forraje verde & 2,26 & 52,03 & $18,85 * * *$ \\
\hline Pienso & 49,08 & 35,20 & $44,46 * * *$ \\
\hline Forraje seco & 17,67 & 9,59 & $14,98 * *$ \\
\hline
\end{tabular}

${ }^{(1)}$ Forraje verde. ${ }^{(2)}$ Superficie Agraria Útil dedicada al ganado de leche. ${ }^{(3)}$ Unidades de Ganado

Mayor. ${ }^{(4)}$ Significación estadística: $* *(\mathrm{p}<0,01)$; *** $(\mathrm{p}<0,001)$.

Fuente: elaboración propia.

\subsection{Relación entre dieta y estructura territorial}

De las 41 variables territoriales, las ganaderías incluidas en el grupo FV presentan diferencias estadísticamente significativas en tan sólo 10 de estas variables respecto a las NFV (Cuadro 2). Tres de las variables guardan relación con la altitud (altura parcela PS, altura parcela PS a $\leq 1 \mathrm{~km}$ y altura parcela TA $\mathrm{a} \leq 1 \mathrm{~km}$ ), cuatro con métricas del paisaje (índice de la mancha PS más grande, índice de forma, porcentaje de superficie de núcleo central de manchas PS sobre superficie total e índice de división del paisaje); siendo las tres restantes variables relacionadas con el uso de la superficie (\% de la superficie total dedicada a pastos y superficie TA declarada leche $a \leq 1 \mathrm{~km}$ ) y la calidad del suelo para uso agrícola (\% PS suelo de cat. A).

Los resultados muestran que la estructura del territorio tiene una menor relevancia en el sistema de alimentación de lo esperado en un principio. Si bien variables directamente relacionadas con la geografía del paisaje (altitud y métricas) se consideran esenciales.

Cuadro 2. Diferencias en algunas de las características territoriales, según tipo de ganadería $F V(\geq 25 \% \mathrm{FV})$ y $N F V(<25 \% \mathrm{FV})$, año 2015

\begin{tabular}{|c|c|c|c|}
\hline \multirow{3}{*}{ Variables territoriales (valores medios) } & Grupo NFV & Grupo FV & \multirow{2}{*}{ Total } \\
\hline & $<25 \% \mathrm{FV}^{(1)}$ & $\geq 25 \% \mathrm{FV}$ & \\
\hline & $\mathrm{N}=66$ & $\mathrm{~N}=33$ & $\mathrm{~N}=99$ \\
\hline Altura parcelas PS ${ }^{(2)}\left(\mathrm{msnm}^{(3)}\right)$ & 145,97 & 302,52 & $198,15 * * *(5)$ \\
\hline Altura parcelas PS a $\leq 1 \mathrm{~km}(\mathrm{msnm})$ & 140,00 & 289,06 & $189,69 * * *$ \\
\hline Altura parcelas TA $\mathrm{a} \leq 1 \mathrm{~km}(\mathrm{msnm})$ & 78,34 & 102,60 & $81,38 *$ \\
\hline$\%$ PS s. superficie total $\left(\mathrm{PS}+\mathrm{TA}^{(4)}\right)$ & 81,37 & 96,37 & $86,37 * *$ \\
\hline Índice de la mancha PS más grande (\%) & 20,23 & 28,26 & $22,90 *$ \\
\hline Valor medio índice de forma & 1,71 & 1,64 & $1,69 *$ \\
\hline \% Superf núcleo central manchas PS s. superf total (PS+TA) & 76,45 & 90,84 & $81,25 * *$ \\
\hline Índice de división del paisaje (\%) & 89,36 & 81,86 & $86,86 *$ \\
\hline Superficie TA declarada leche a $\leq 1 \mathrm{~km}$ (ha) & 18,18 & 4,32 & $13,56 * *$ \\
\hline \% PS suelo categoría A & 12,65 & 4,27 & $9,86 *$ \\
\hline
\end{tabular}

${ }^{(1)}$ Forraje verde. ${ }^{(2)}$ PS: Pastos permanentes. ${ }^{(3)} \mathrm{msnm}$ : metros sobre el nivel del mar. ${ }^{(4)} \mathrm{TA}$ : Tierras arables.

${ }^{(5)}$ Significación estadística: ns (no significativa); * $(\mathrm{p}<0,05)$; **(p<0,01); *** $(\mathrm{p}<0,001)$.

Fuente: elaboración propia.

Una de las diferencias más notables entre las ganaderías es la altitud; en las ganaderías del grupo FV la altura media de las parcelas PS (tanto en el conjunto de la superficie de la explotación como a $\leq 1 \mathrm{~km}$ ), y las TA a $\leq 1 \mathrm{~km}$ de la ganadería, es mayor que en el grupo NFV. Esto indica que estas ganaderías NFV, más intensivas y más grandes, se sitúan en zonas más mecanizables. 
El porcentaje de superficie dedicada a pastos (PS) sobre la superficie total es también mayor en el grupo FV, lo cual está ligado a la mayor dedicación en las ganaderías NFV al cultivo de maíz (relacionado con los datos de silo de maíz del Cuadro 1).

Las ganaderías FV presentan un menor valor medio del índice de forma que NFV, lo cual indica una mejor geometría de las parcelas. En las FV también es más bajo el índice de división del paisaje, esto es, la menor probabilidad de que dos lugares del paisaje, elegidos al azar, no correspondan, los dos, a la misma mancha PS; este índice es mejor cuanto más bajo. Por el contrario, el índice de mancha PS más grande sobre la superficie total es más elevado en FV lo que significa que es mayor el porcentaje que representa la mayor de las manchas de tipo PS con respecto a la superficie total (PS+TA) de la explotación; así pues la mayor dimensión de las manchas en explotaciones FV puede estar detrás de su mejor geometría.

Con respecto a la calidad del suelo, se observa que las ganaderías FV tienen un suelo con menor capacidad de uso agrícola. Las parcelas gestionadas por estas ganaderías FV están localizadas en zonas de mayor altitud, apartadas de la costa y con mayor pendiente, normalmente asociadas al aprovechamiento para pastos.

\section{Conclusiones}

En primer lugar, cabe indicar que la fijación del umbral del $25 \% \mathrm{FV}$ sobre la materia seca en la dieta de primavera comporta, como no podía ser de otra manera, diferencias en el manejo alimentario y en las características productivas. En este sentido, las ganaderías FV tienen menor dimensión territorial, rebaño, carga ganadera, producción anual de leche y menor utilización de concentrado, mientras que destinan más horas al pastoreo, contrariamente a las características de las ganaderías NFV.

En segundo lugar, las ganaderías FV muestran diferencias en la estructura territorial respecto a las ganaderías NFV, pero menos marcadas de lo esperable. Esto puede ser debido a que la agrupación de las ganaderías no se ha hecho en función de si se da o no forraje verde a las vacas en lactación sino en función del porcentaje de forraje verde suministrado (marcando un umbral del 25\%), pues el objeto era la estimación del potencial territorial para producir leche de pasto, definida arbitrariamente de esa manera, cuando quizá es el empleo o no de forraje verde el que marca las diferencias más acusadas en el manejo territorial.

En último lugar, apuntar que los resultados presentados son la base previa de un análisis posterior sobre el potencial territorial de la producción de leche en base a pasto en Cantabria, al tiempo que plantean un camino de investigación futura. Con ello se pretende contribuir al estudio de su estructura productiva, como estrategia de valorización de los sistemas extensivos de producción de leche ligada al territorio.

\section{Bibliografía}

García, E., Ruiz, F., García, A.I. y Vázquez, I. (2020). “Caracterización productiva y socioeconómica de las explotaciones con vacas de carne en Cantabria”. REEAP, 256: 175-196.

Huyghe, C., De Vlieger, A., Van Gils, B. y Peeters, A. (2014). Grassland and hebivore production in Europe and effects of common policies. Éditions Quae. Versailles.

MAPA (2020). Estructura del sector vacuno lechero en España 2016-2020. Subdirección General de Producciones Ganaderas y Cinegéticas, Dirección General de Producciones y Mercados Agrarios. https://www.mapa.gob.es/es/ganaderia/temas/produccion-y-mercadosganaderos/estructurasectorvacunolechero2016-2020def_tcm30-540399.pdf. [consulta 05/21].

McGarigal, K. (2017). Landscape metrics for categorical map patterns. Lecture Notes. Available online: http://www.umass.edu/landeco/teaching/landscape_ecology/schedule/chapter9 metrics.pdf.

NRC (2001). National Research Council. Nutrient Requirements of Dairy Cattle, 7th ed. National Academies Press: Washington, DC.

Smit, H.J., Metzger, M.J. and Ewert, F. (2008). "Spatial distribution of grassland productivity and land use in Europe". Agricultural Systems, 98: 208-2019.

Villar, A., Vázquez-González, I., Vicente, F., Salcedo, G., González, L., Botana, A., Royo, L.J., Eguinoa, P. y Busqué, J. (2021a). "Study of the variability in fatty acids and carotenoid profiles: laying the ground for tank milk authentication”. Sustainability, 13 (8): 4506. https://www.mdpi.com/20711050/13/8/4506.

Villar, A., Salcedo, G., Vázquez, I., Suárez, E. y Busqué J. (2021b). "Is it possible to estimate the composition of a cow's diet based on the parameters of its milk?" Sustainability, 13 (8): 4474. https://www.mdpi.com/2071-1050/13/8/4474.

CIFA (2007). Los pastos en Cantabria y su aprovechamiento. https://cifacantabria.org/Documentos/memoria.pdf. 